\title{
Endoscopic Removal of a Bullet Penetrating the Middle Cranial Fossa
}

\author{
Neal U. Hatch, B.S., ${ }^{1}$ Kristen O. Riley, M.D., ${ }^{2}$ and Bradford A. Woodworth, M.D. ${ }^{1}$
}

Reports of intracranial retained foreign bodies are relatively rare in the literature. Such objects can cause numerous complications requiring removal, such as infection, persistent cerebrospinal fluid (CSF) leak, or new-onset seizures. The transnasal endoscopic approach provides an excellent alternative to craniotomy for repairing middle cranial fossa (MCF) defects. We describe a case of a 57-year-old woman with a self-inflicted bullet piercing the MCF, creating a persistent CSF leak. The details regarding the removal of this penetrating foreign body from the $\mathrm{MCF}$, including the unique management in the setting of a contralateral spontaneous CSF leak, are discussed.

KEYWORDS: Middle cranial fossa, foreign body, skull base surgery, endoscopic approach

Transnasal endoscopic surgical removal of foreign bodies that transgress the anterior skull base has become a more attractive option when compared with traditional open surgical methods. Advancements in endoscopic surgical technology and techniques have provided superb access to the skull base, allowing removal of objects that normally would require a craniotomy-a procedure with significant morbidity and mortality. ${ }^{1}$ The decision to leave a foreign body in place is often based on its location near vital structures such as the internal carotid artery. Preoperative imaging is critical for operative planning and should be used to determine the exact location of the object as well as to anticipate the appropriate surgical access required for removal. The decision to remove retained intracranial foreign bodies is based on prevention of potential complications. Objects penetrating the sterile dural environment may result in high risk of central nervous system infection and cerebrospinal fluid (CSF) leak. ${ }^{2}$ Other reported complications of retained intracranial foreign bodies include carotid artery occlusion without neurological sequelae, ${ }^{3}$ new-onset seizures with foreign body reaction, ${ }^{4}$ hydrocephalus, ${ }^{5}$ nerve and vessel injury due to object migration, ${ }^{6}$ and lead poisoning. ${ }^{7} \mathrm{We}$ present our unique management of a persistent CSF leak caused by a middle cranial fossa (MCF) bullet in the setting of a discrete, contralateral spontaneous CSF leak resulting from intracranial hypertension.

\section{CASE REPORT}

A 57-year-old obese (body mass index: 38.3) woman with a self-inflicted gunshot wound to the face presented to an outside institution. The bullet, entering the left face, penetrated the floor of the MCF in the left sphenoid bone. CSF leaks were repaired via two separate frontal craniotomies using a pericranial flap to repair a comminuted anterior skull base. She was subsequently referred to our department for management of a persistent CSF leak. Physical exam was significant for poor
${ }^{1}$ Division of Otolaryngology-Head and Neck Surgery, Department of Surgery; ${ }^{2}$ Division of Neurosurgery, University of Alabama at Birmingham, Birmingham, Alabama.

Address for correspondence and reprint requests: Bradford A. Woodworth, M.D., BDB 563, 1530 3rd Ave. S, Birmingham, AL 35294 (e-mail: bwoodwo@hotmail.com).

Skull Base Rep 2011;1:47-50. Copyright (C) 2011 by Thieme
Medical Publishers, Inc., 333 Seventh Avenue, New York, NY 10001, USA. Tel: +1(212) 584-4662.

Received: August 9, 2010. Accepted: January 24, 2011. Published online: March 30, 2011.

DOI: http://dx.doi.org/10.1055/s-0031-1275633.

ISSN 2157-6971. 


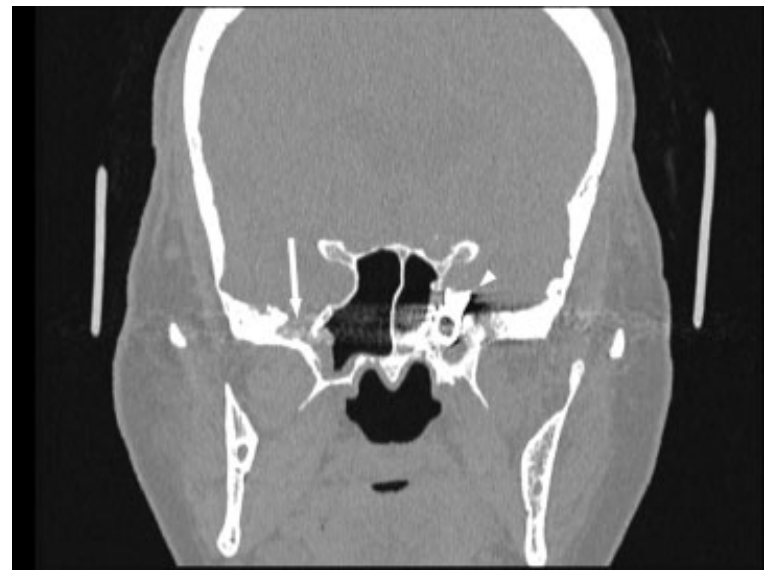

Figure 1 Coronal computed tomography scan reveals a bullet (arrowhead) penetrating the middle cranial fossa through the left lateral recess of the sphenoid sinus. A contralateral defect is identified with multiple arachnoid pits present (arrow).

visual acuity and complete numbness in the distribution of the left maxillary portion of the trigeminal nerve. Nasal endoscopy examination revealed little evidence of trauma to the nasal cavity. Clear drainage was visible with Valsalva in the region of the left middle meatus and sphenoethmoidal recess. Computed tomography (CT) scan demonstrated severe trauma to the left anterior skull base involving the left cribriform plate, ethmoid sinus, orbital roof, and lateral wall of the left sphenoid sinus. A retained bullet was visualized within the left sphenoid bone and clivus. There were prominent arachnoid granulations in bilateral MCFs, indicating a possible history of increased intracranial pressure (ICP). ${ }^{8}$ A small skull base defect was identified preoperatively in the floor of the right $\mathrm{MCF}$, representing a possible synchronous spontaneous CSF leak (Fig. 1). Because the bullet bordered the left internal carotid artery, a diagnostic cerebral angiogram was performed showing complete occlusion of the left internal carotid artery. No neurological sequelae were noted on preoperative examination.

\section{SURGICAL PROCEDURE}

Instillation of fluorescein via a lumbar drain was performed as previously described. ${ }^{9}$ Access to the sphenoid sinus was obtained endoscopically via a transpterygoid approach. Following a complete ethmoidectomy, the left sphenoid sinus was opened and dissection was carried back into the pterygopalatine fossa, where the internal maxillary artery was clipped. The pterygoid plates were drilled and opened up widely, exposing the bullet. This revealed copious fluorescein-stained CSF leaking in this region (Figs. 2 and 3). The right side was also examined because a defect was identified on the preoperative CT scan. A small encephalocele with a concurrent CSF leak was noted in the right lateral recess (Fig. 4). After extensive dissection, the bullet casing and bullet were removed as separate pieces, taking care not to damage the involved brain parenchyma. Any visualized implanted mucosa in the MCF was removed and the defect was closed with Surgisis ${ }^{\circledR}$ (Cook Medical, Bloomington, IN) as a dural substitute and supported with abdominal fat. Because of the large size of the defect, a pedicled septal mucosal flap was also created from the right side of the septum to cover the entire obliterated lateral recess (Fig. 4). Because the internal maxillary artery was ligated as part of the surgical approach, the left septal mucosa could not be used. An intraoperative decision was made to delay closure of the right side because a transpterygoid approach on the right would require ligation of the internal maxillary artery and compromise the blood supply to the septal flap. Lumbar pressure was measured according to previously established protocol on postoperative day 2 and determined to be $32 \mathrm{~cm} \mathrm{H}_{2} \mathrm{O}$ pressure. Due to the increased CSF leak recurrence rate demonstrated in patients with concurrent elevated $\mathrm{ICP}^{9,10}$ and

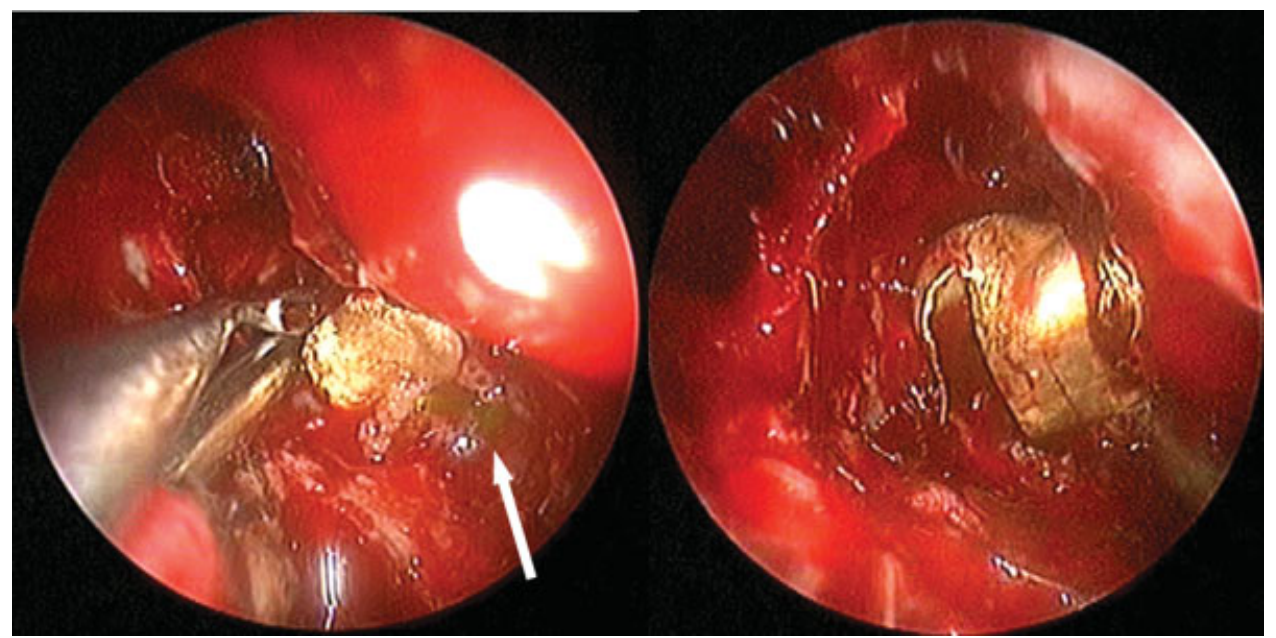

Figure 2 The bullet casing is removed following exposure of the lateral recess through a transpterygoid approach. Note the green staining of the fluorescein (arrow). 


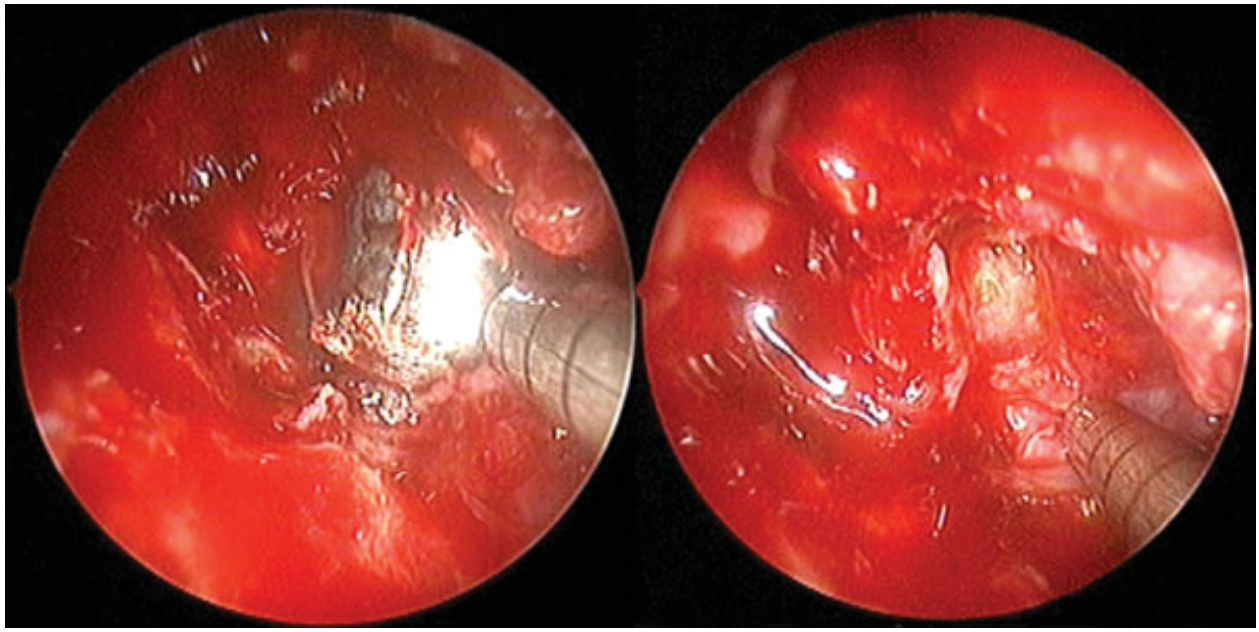

Figure 3 The bullet is removed from the middle cranial fossa (left), leaving a large skull base defect (right).

in the setting of a contralateral CSF leak that could not be repaired at the time of surgery, a ventriculoperitoneal (VP) shunt was performed. ${ }^{11}$

The patient had an uneventful hospital course and was discharged for a planned second-stage transpterygoid approach to the contralateral encephalocele at 8 weeks. The purpose of surgical delay was to allow the septal flap to heal and avoid compromised blood flow from ligating the left internal maxillary artery during the transpterygoid approach. However, her right-sided CSF rhinorrhea stopped upon placement of the VP shunt and the patient decided to postpone her surgery. She continues to have no evidence of a CSF leak at 6 months of clinical follow-up.

\section{DISCUSSION}

Retained intracranial foreign bodies result from iatrogenic injury, self-inflicted harm, or accidental trauma. In other cases, the retained foreign body may be incidentally found (i.e., after remote trauma) due to complications such as new-onset seizures. ${ }^{4}$ Those with foreign body trauma to the orbit or sinonasal region may have unsuspected intracranial penetration of the skull base. Thus, imaging is crucial for determining location of penetration as well as proximity to brain parenchyma and important vascular structures. Imaging can also have added utility in the identification of unknown objects by their physical characteristics on CT and magnetic resonance (MR). Metal and graphite objects may be recognized by their hyperattenuated appearance on $\mathrm{CT} .{ }^{4}$ Other materials such as wood or plastic can be variable-hyperdense or hypodense relative to fat for plastic and even mimicking air as is the case for wood. ${ }^{12,13}$ Contrast enhancement can be useful in differentiating foreign bodies from important vascular structures. ${ }^{12} \mathrm{MR}$ can be complementary to CT but should never be performed when the object is suspected to be metallic as this could cause expulsion of the object by the magnet.

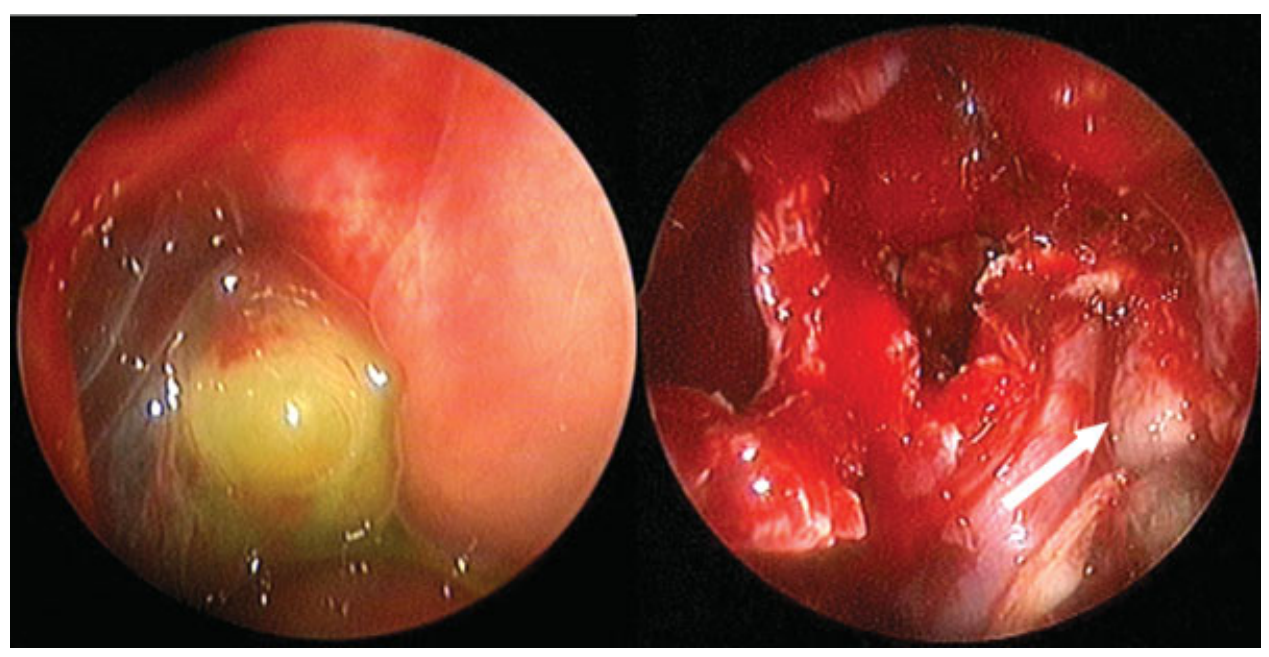

Figure 4 A contralateral encephalocele is identified using 70-degree nasal endoscopy (left). A septal flap (arrow) elevated from the right side of the septum is used to seal off the left lateral recess of the sphenoid sinus (right). 
The decision to use the endoscopic approach depends upon location, skill of the surgeon, and availability of neurosurgical assistance. ${ }^{14}$ Because of the complex anatomy and important vascular structures, particularly in the MCF, preoperative imaging with angiography is crucial for operative planning if there is proximity to the cerebral vasculature. Our patient was deemed a low risk of hemorrhage secondary to occlusion of the left carotid demonstrated with angiography. Objects located near the internal carotid or cerebral arteries pose a significant risk of uncontrollable hemorrhage and vascular injury. Neurosurgery conversion to craniotomy would be necessary in cases of poor visualization or difficulty with hemostasis. If there is high risk of uncontrollable hemorrhage, craniotomy would be the preferred initial choice for removal of the object.

Endoscopic repair of CSF leaks has been demonstrated to be highly effective in preventing primary and recurrent cases of meningitis. ${ }^{11} \mathrm{~A}$ successful repair rate of $\sim 90 \%$ is seen in most series, demonstrating the effectiveness of endoscopic repair. ${ }^{9,11}$ Multiple studies have shown the type of tissue used (i.e., fat, fascia, acellular collagen, and pericranium) to repair leaks does not influence recurrence rates. ${ }^{11}$ Pedicled septal flaps are also useful for large defects and facilitate rapid healing and closure ${ }^{15}$ Ligation of the internal maxillary artery is necessary during the transpterygoid approach to the lateral recess of the sphenoid sinus. This artery provides the blood supply to the septal flap and presented a surgical dilemma in the present case. In the setting of the contralateral spontaneous CSF leak that would require a similar transpterygoid approach, we chose to delay the surgery to avoid compromised blood supply to the flap.

Spontaneous CSF leaks with concurrent increased ICP have been associated with higher leak recurrence rates. ${ }^{11}$ However, treatment of increased ICP in conjunction with endoscopic repair produces success rates (95\%) similar to that of other CSF leak etiologies. ${ }^{9}$ In the present case, the patient's CSF rhinorrhea stopped by controlling the underlying pathophysiology (intracranial hypertension) with insertion of a VP shunt.

\section{CONCLUSION}

Transnasal endoscopic techniques remain a highly effective means of repairing skull base defects. Access to an
MCF foreign body via a transpterygoid approach was an appropriate choice for surgical removal in this patient and further expands the indications for endoscopic skull base approaches. In select patients, endoscopic removal of retained intracranial foreign bodies with adequate repair of associated CSF leaks can be achieved.

\section{REFERENCES}

1. Ganly I, Patel SG, Singh B, et al. Complications of craniofacial resection for malignant tumors of the skull base: report of an International Collaborative Study. Head Neck 2005;27:445-451

2. Sharif S, Roberts G, Phillips J. Transnasal penetrating brain injury with a ball-pen. Br J Neurosurg 2000;14:159-160

3. Loes DJ, Smoker WR, Menezes AH. Pen injury: a rare complication. Pediatr Neurol 1987;3:44-47

4. Aulino JM, Gyure KA, Morton A, Cole JW. Temporal lobe intraparenchymal retained foreign body from remote orbital trauma. AJNR Am J Neuroradiol 2005;26:1855-1857

5. Dodson KM, Bridges MA, Reiter ER. Endoscopic transnasal management of intracranial foreign bodies. Arch Otolaryngol Head Neck Surg 2004;130:985-988

6. Rengachary SS, Carey M, Templer J. The sinking bullet. Neurosurgery 1992;30:291-294; discussion 294-295

7. Kikano GE, Stange KC. Lead poisoning in a child after a gunshot injury. J Fam Pract 1992;34:498-500, 502, 504

8. Barañano CF, Curé J, Palmer JN, Woodworth BA. Sternberg's canal: fact or fiction? Am J Rhinol Allergy 2009;23:167-171

9. Woodworth BA, Prince A, Chiu AG, et al. Spontaneous CSF leaks: a paradigm for definitive repair and management of intracranial hypertension. Otolaryngol Head Neck Surg 2008;138:715-720

10. Woodworth BA, Palmer JN. Spontaneous cerebrospinal fluid leaks. Curr Opin Otolaryngol Head Neck Surg 2009;17:59-65

11. Banks CA, Palmer JN, Chiu AG, O'Malley BW Jr, Woodworth BA, Kennedy DW. Endoscopic closure of CSF rhinorrhea: 193 cases over 21 years. Otolaryngol Head Neck Surg 2009;140:826-833

12. Matsumoto S, Hasuo K, Mizushima A, et al. Intracranial penetrating injuries via the optic canal. AJNR Am J Neuroradiol 1998;19:1163-1165

13. Adesanya OO, Dawkins DM. Intraorbital wooden foreign body (IOFB): mimicking air on CT. Emerg Radiol 2007;14: 45-49

14. Thomas S, Daudia A, Jones NS. Endoscopic removal of foreign body from the anterior cranial fossa. J Laryngol Otol 2007;121:794-795

15. Hadad G, Bassagasteguy L, Carrau RL, et al. A novel reconstructive technique after endoscopic expanded endonasal approaches: vascular pedicle nasoseptal flap. Laryngoscope 2006;116:1882-1886 